\title{
Dressed Ion-Pair States of an Ultralong-Range Rydberg Molecule
}

\author{
P. Giannakeas $\odot,{ }^{1, *}$ Matthew T. Eiles $\odot,{ }^{1, \dagger}$ F. Robicheaux $\odot,{ }^{2,3, \hbar}$ and Jan M. Rost ${ }^{1, \S}$ \\ ${ }^{1}$ Max-Planck-Institut für Physik komplexer Systeme, Nöthnitzer Street 38, D-01187 Dresden, Germany \\ ${ }^{2}$ Department of Physics and Astronomy, Purdue University, West Lafayette, Indiana 47907, USA \\ ${ }^{3}$ Purdue Quantum Science and Engineering Institute, Purdue University, West Lafayette, Indiana 47907, USA
}

(Received 22 May 2020; accepted 21 August 2020; published 16 September 2020)

\begin{abstract}
We predict the existence of a universal class of ultralong-range Rydberg molecular states whose vibrational spectra form trimmed Rydberg series. A dressed ion-pair model captures the physical origin of these exotic molecules, accurately predicts their properties, and reveals features of ultralong-range Rydberg molecules and heavy Rydberg states with a surprisingly small Rydberg constant. The latter is determined by the small effective charge of the dressed anion, which outweighs the contribution of the molecule's large reduced mass. This renders these molecules the only known few-body systems to have a Rydberg constant smaller than $R_{\infty} / 2$.
\end{abstract}

DOI: 10.1103/PhysRevLett.125.123401

The richness of Rydberg physics is highlighted by two exotic molecular systems which have attracted recent interest: ultralong-range Rydberg molecules (ULRM) and heavy Rydberg states (HRS). The ULRM is a fragile dimer with a bond length $\sim 100$ nanometers. This gargantuan molecule consists of a neutral perturber atom $(B)$ bound to a highly excited Rydberg atom $\left(A^{*}\right)$ [1-4]. The experimental observation of ULRMs [5-7] has led to their use in many diverse applications, e.g., as probes of chargeneutral interactions [8-13] or as impurities embedded in a many-body bath [14-24]. ULRMs exist because the Rydberg electron accumulates an appreciable phase shift as it scatters off of the perturber, which in turn produces an energy shift proportional to the $S$-wave scattering length $[25,26]$. This binds the "trilobite" molecule, $A^{*} B$, together [27-29]. If the electron-perturber $(e-B)$ complex possesses a $P$-wave shape resonance, a second, more deeply bound, "butterfly" ULRM forms [30-32].

HRS (also called ion pairs or heavy Bohr atoms) are the direct molecular analogs of a Rydberg atom [33-37]. An atomic anion replaces the Rydberg electron, creating a vibrational Rydberg state $A^{+} B^{-}$. The properties of these molecules obey typical Rydberg formulae, but with the electron's mass replaced by the dimer's heavy reduced mass. Typically the excitation of pairs of ground state atoms to HRS is difficult due to weak Franck-Condon factors and electronic transition-dipole moments [36,38].

Published by the American Physical Society under the terms of the Creative Commons Attribution 4.0 International license. Further distribution of this work must maintain attribution to the author(s) and the published article's title, journal citation, and DOI. Open access publication funded by the Max Planck Society.
Recent proposals exploit ULRMs, with similar bond lengths as HRS, to avoid these challenges $[39,40]$. In the vicinity of the perturber, the electronic wave function of the butterfly molecule and the metastable excited $P$ anion have the same symmetry [9]. The electron can thus be transferred from the Rydberg state into the bound $S$ anion state via a dipole-allowed transition, which also supplies the required energy to match the electron affinity and allow the reaction $A^{*} B \rightarrow A^{+} B^{-}$to occur.

However, the exotic systems of ULRMs and HRS exist typically in well separated energy intervals. In this Letter, we predict a class of highly excited molecular states which realize properties of HRS on the electronic energy scale of ULRMs, thus combining both concepts. The inclusion of higher partial waves $(L \geq 2)$ in the $e-B$ interaction yields a hierarchy of "truncated Coulomb" potential energy curves (PECs) governing the vibrational motion associated with every degenerate electronic Rydberg manifold, labeled by $n$. Each level in the infinite electronic Rydberg series becomes the dissociation threshold for a set of trimmed heavy Rydberg series, or THRS [see Fig. 1(c)]. The tHRS possess only a finite number of vibrational states since the "truncated Coulomb" PECs vanish once the perturber resides outside the Rydberg electron's orbit. The THRS preserve the basic attributes of ULRMs; in particular, the perturbed electronic wave function fills the entire Rydberg volume, while in HRS the electron binds to the perturber. To address the physical origin of the THRS, we map the ULRM system, $A^{*} B$ [upper panel of Fig. 1(d)] onto an effective ion pair, $A^{+} B^{Q}$ [lower panel of Fig. 1(d)] where the perturber is dressed by a fractional charge $Q$.

The dressed ion-pair model reveals that the perturberinduced localization of the electron yields an effective negative charge which is virtually independent of the 


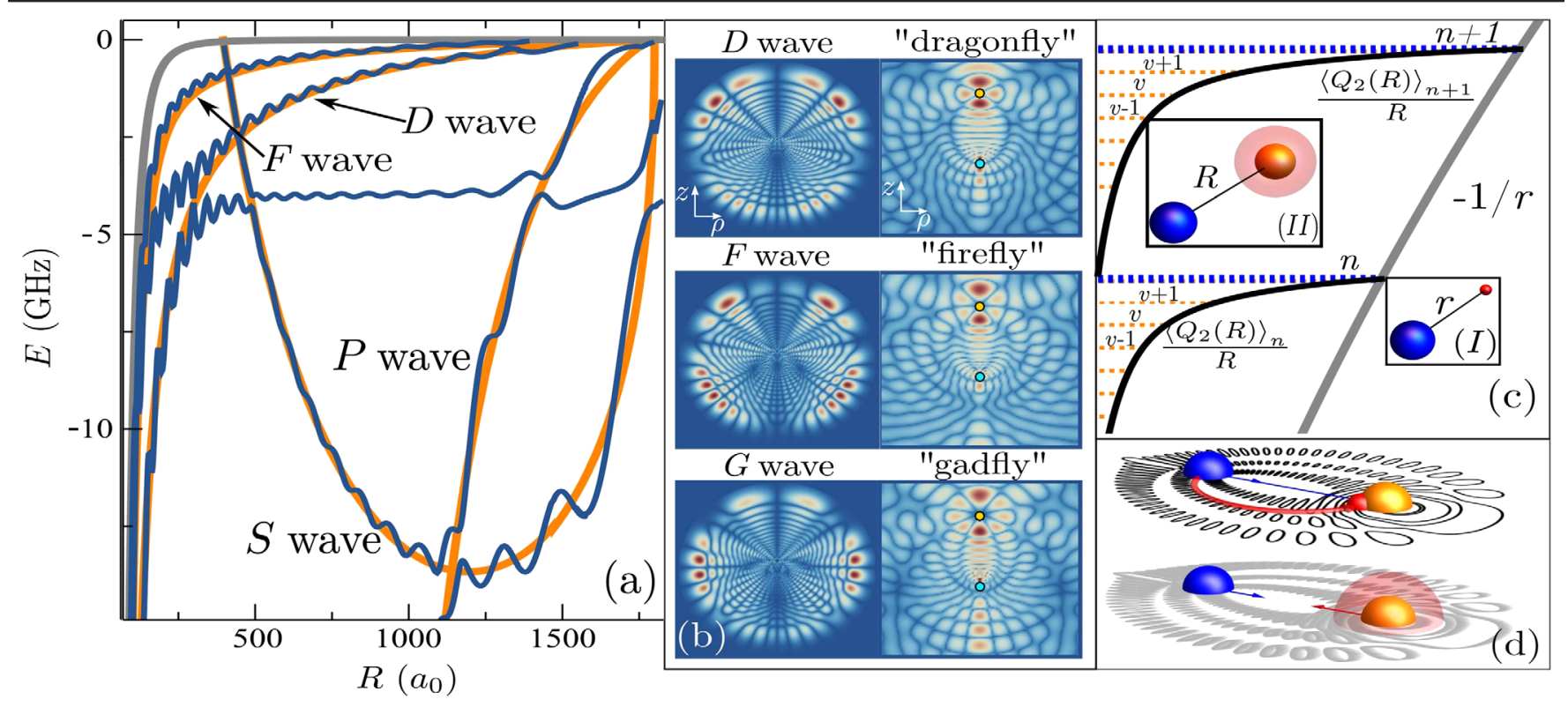

FIG. 1. (a) ${ }^{\Sigma} \mathrm{Rb}_{2}$ Rydberg molecule PECs (blue) relative to the $n=30$ manifold. The smooth orange curves overlaid are the results of the model, Eq. (2), and the ion-atom polarization potential is shown in gray. (b) Electronic wave functions $\Psi_{\Sigma}(z, \rho)$ in cylindrical coordinates are depicted for the family of $L \geq 2$ states. The left column shows $\rho \sqrt{\left|\Psi_{\Sigma}(z, \rho)\right|}$ for $z \in[-2000,2000]$ and $\rho \in[-2000,2000]$. The right column shows $\sqrt{\left|\Psi_{\Sigma}(z, \rho)\right|}$ for $z \in[-250,300]$ and $\rho \in[-250,250]$. The blue (orange) dot indicates the position of the Rydberg core (perturber). (c) A schematic of the vibrational Rydberg series. The electron-ion potential (gray) supports the bound states of the Rydberg atom (I). Each electronic Rydberg manifold labeled $n, n+1, \ldots$ (blue dashed lines) is the threshold for the $M=2 D$-wave PEC (black). This PEC supports a nuclear Rydberg series (orange dotted lines) of dressed ion-pair states (II) labeled $v-1, v, \ldots$ (d) Two pictures of a ULRM: on top, the typical picture, where the Rydberg electron (red) scatters from the perturber (orange) and forms a trilobitelike wave function (black contour). On the bottom, the dressed ion-pair picture, wherein the Rydberg wave function is neglected and the relevant quantity is the electron's charge distribution (red) around the perturber, forming a dressed anion.

internuclear distance $R$, leading thus to Coulomb-like molecular PECs. This property arises due to the linear energy dependence of the electron-perturber phase shifts for $L \geq 2$ which is universally satisfied for any type of polarizable perturber at low energies. Additionally, the magnitude of the fractional charge counterbalances the heavy mass of the THRS and leads to a Rydberg constant which is significantly smaller than $R_{\infty} / 2$, the Rydberg constant of positronium, the lightest ion pair.

We compute the ULRM PECs including $L \geq 2$ phase shifts by employing the generalized local frame transformation (GLFT) approach [41]. This framework requires as input only a set of atomic quantum defects $\mu_{l}$ and scattering phase shifts $\delta_{L}$ with $l(L)$ indicating the electron's angular momentum relative to $A^{+}(B)$. The generic scope of the GLFT approach permits us to include the effect of higher partial wave $e-B$ scattering, avoiding the limitations of the Fermi-Omont pseudopotential or Green's function methods $[3,25,26,42-46]$. The triplet $e-B$ scattering phase shifts are obtained from a nonrelativistic two-electron $R$-matrix code [47,48]. We consider $\mathrm{Rb}$ atoms and use atomic units unless otherwise specified. To emphasize the universal aspects of the THRS, we neglect the nuclear and electronic spin degrees-of-freedom, ignore spin-orbit and hyperfine structure effects, and use only the triplet $e-B$ phase shifts. Such a scenario is realizable in a spin-stretched experiment. $M$, the projection of $L$ onto the internuclear axis $R$, is a good quantum number and defines the molecular symmetry.

Figure 1(a) shows the $\Sigma(M=0) \mathrm{Rb}_{2}$ PECs relative to the $n=30$ Rydberg manifold including $L \leq 3$ partial waves. The blue curves depict the trilobite PEC ( $S$-wave scattering), the butterfly PEC ( $P$ wave), as well as a new series of $L \geq 2$ PECs which complete the ULRM "family": the "dragonfly" PEC ( $D$ wave), "firefly" PEC ( $F$ wave), and so on. As $L$ increases the PECs condense into the ion-atom potential $-\alpha / 2 R^{4}$ (gray curve), where $\alpha$ is the atomic polarizability. Exemplary electronic wave functions with the perturber placed at $R=200 a_{0}$ are displayed in Fig. 1(b). Near the perturber they manifest the dominant spherical symmetry of the $e-B$ interaction since the Coulomb field is negligible. Thus, $L$ is approximately a good quantum number for labeling these states. These molecules exhibit dipole moments in the hundreds of debye, similar to the trilobite and butterfly molecules (see the Supplemental Material [49]).

Unlike the trilobite and butterfly PECs, the $L \geq 2$ PECs are, to a good approximation, Coulombic. This is particularly evident for molecular states having higher $M$ values, since the oscillatory fringes in the PECs vanish for 
increasing $M$. For example, Fig. 1(c) schematically depicts in black the $\Delta(M=2)$ dragonfly PECs, which dissociate at each electronic Rydberg $n$ manifold (blue dotted lines).

The dressed ion-pair model captures intuitively the emergence of the Coulombic character in the $L \geq 2$ PECs, simultaneously illustrating why the $S$ - and $P$-wave PECs are so different. The standard depiction of an ULRM, for example a trilobite, is depicted in the upper panel of Fig. 1(d). The perturber mixes the degenerate Rydberg states to form the trilobite wave function, plotted as a contour. The nodal pattern of this electronic wave function is linked to the oscillatory fringes of the $S$-wave PEC [see Fig. 1(a)]. Starting out tabula rasa, in the bottom panel of Fig. 1(d) the ULRM is viewed as an effective ion pair where the perturber is dressed by a charge distribution [red sphere in Fig. 1(d)] forming an anion of fractional charge. This perspective ignores the total trilobite wave function except for the charge distribution localized by the $e-B$ interaction.

The effective charge is obtained by calculating the difference in electronic probability accumulated in the vicinity of the perturber with and without its presence. The resulting integral is evaluated in terms of the phase shift $\delta_{L}(k)$ at a given electronic scattering kinetic energy $\left[k_{n}(R)\right]^{2} / 2=1 / R-1 / 2 n^{2}$. The charge distribution at the perturber is

$$
\left\langle Q_{L}(R)\right\rangle_{n}=-\left.\frac{\left(\frac{d \nu}{d E}\right)^{-1}}{\pi k_{n}(R)} \frac{d \delta_{L}(\kappa)}{d \kappa}\right|_{\kappa=k_{n}(R)},
$$

where $\nu$ is related to the electronic energy $E$ via $E=-1 / 2 \nu^{2}$. The presence of the Rydberg electron's density of states $d \nu / d E$ arises from the quantization of the $e-B$ scattering continuum by the Coulomb field of $A^{*}$ (for details see [49]). The charge in Eq. (1) is proportional to the time delay of the $e-B$ subsystem, which leads to a transparent interpretation [51]. A large and positive time delay implies that the electron slows down near the perturber, which consequently appears to be dressed with a negative charge. A negative time delay has the opposite consequence: the electron spends less time near the perturber than elsewhere, dressing it with a positive charge.

The dressed anion and the positively charged Rydberg core interact via a Coulomb force, $F_{L}(R)=\left\langle Q_{L}(R)\right\rangle_{n} / R^{2}$, yielding a potential energy $V_{L}(R)$ relative to the $n$th manifold,

$V_{L}(R)=\frac{1}{2 n^{2}}-\frac{1}{2\left(n-\delta_{L}\left[k_{n}(R)\right] / \pi\right)^{2}}, \quad$ for $R \leq R_{c}$,

where $V_{L}\left(R>R_{c}\right)=0$ for a perturber located outside the electron's orbit $\left(R_{c}=2 n^{2}\right)$. Note that Eq. (2) was also obtained via different methods in Ref. [52], which emphasized its similarities with the Rydberg formula. This highlights that the phase shifts play the role of molecular quantum defects [53]. Due to the semiclassical nature of the dressed ion-pair model, Eq. (2) captures only the shape of the molecular PECs [see orange lines in Fig. 1(a)].

The effective charge in Eq. (1) elucidates the emergence of Coulombic molecular PECs. For a generic phase shift $\delta_{L}$, as for $S$ or $P$ partial waves, the charge fluctuates dramatically as $R$ changes, yielding non-Coulombic PECs. However, at low-energies and for $L \geq 2$, only the centrifugal barrier and the tail of the polarization potential contribute to the phase shifts, imparting on them a universal linear energy dependence. Namely, within the Born approximation (BA), the $L \geq 2$ phase shift is

$$
\delta_{L} \approx \pi \bar{\alpha}_{L} k^{2} ; \quad \bar{\alpha}_{L}=\frac{\alpha}{\left(4 L^{2}-1\right)(2 L+3)} .
$$

We confirmed that this closely matches the calculated phase shifts for the alkali atoms (see [49]). The linear energy dependence yields an effective charge virtually independent of $R$ which imprints the Coulombic character onto the corresponding PECs. Indeed, substituting the BA phase shifts in Eq. (2) and expanding it in powers of $n^{-1}$ yields the corresponding Coulomb potential and additional higher order terms,

$$
U_{L}(R)=-\frac{\alpha}{2 R^{4}}+\frac{\bar{\alpha}_{L}}{n^{5}}-\frac{2 \bar{\alpha}_{L}}{n^{3} R}-\frac{6 \bar{\alpha}_{L}^{2}}{n^{4} R^{2}}+, \ldots,
$$

where in $U_{L}(R)$ the ion-atom polarization potential $-\alpha / 2 R^{4}$ is added. The prefactor of the Coulomb term matches Eq. (1) in the large $n$ limit where $\nu \rightarrow n$, i.e., $\left\langle Q_{L}(R)_{n}\right\rangle=-2 \bar{\alpha}_{L} / n^{3}$, and for $\mathrm{Rb}$ the $L=2$ fractional charge is $\sim-6.08 / n^{3}$. Considering only the dominant Coulomb and constant terms in Eq. (4) results in a nuclear vibrational spectrum which obeys a Rydberg formula $E_{v J}^{n L}$ with a redefined Rydberg constant $R_{n L}{ }^{\prime}$,

$$
E_{v J}^{n L}=\frac{\bar{\alpha}_{L}}{n^{5}}-\frac{R_{n L}^{\prime}}{\left(v-\eta_{J}\right)^{2}} ; \quad R_{n L}^{\prime}=\frac{2 m_{A B} \bar{\alpha}_{L}^{2}}{n^{6}} .
$$

$m_{A B}$ is the reduced mass of the molecule and $J$ is the nuclear angular momentum. The constant energy shift, $\bar{\alpha}_{L} / n^{5}$, included in this formula is consistent with the truncation of the Rydberg series to a finite number of states by the vanishing of $U_{L}(R)$ at the classical turning point. The maximum number of states is given by $v_{\max } \approx \sqrt{2 \bar{\alpha}_{L} m_{A B} / n}$. The nuclear quantum defect $\eta_{J}$ accounts for the effects of the non-Coulombic terms in Eq. (4) as well as the complicated molecular potential energy curves at short internuclear distances, $R<30 a_{0}$. The nuclear defects $\eta_{J}$ are system dependent and their evaluation is beyond the scope of this study.

Figure 2 shows the family of $L \geq 2$ PECs relative to the $n=30$ manifold for (a) $\Sigma(M=0)$ and (b) $\Delta(M=2)$ molecular symmetry, where for simplicity we use the BA phase shifts and set $\mu_{l}=0$. In both panels the PECs within the dressed ion-pair model (orange) condense to 


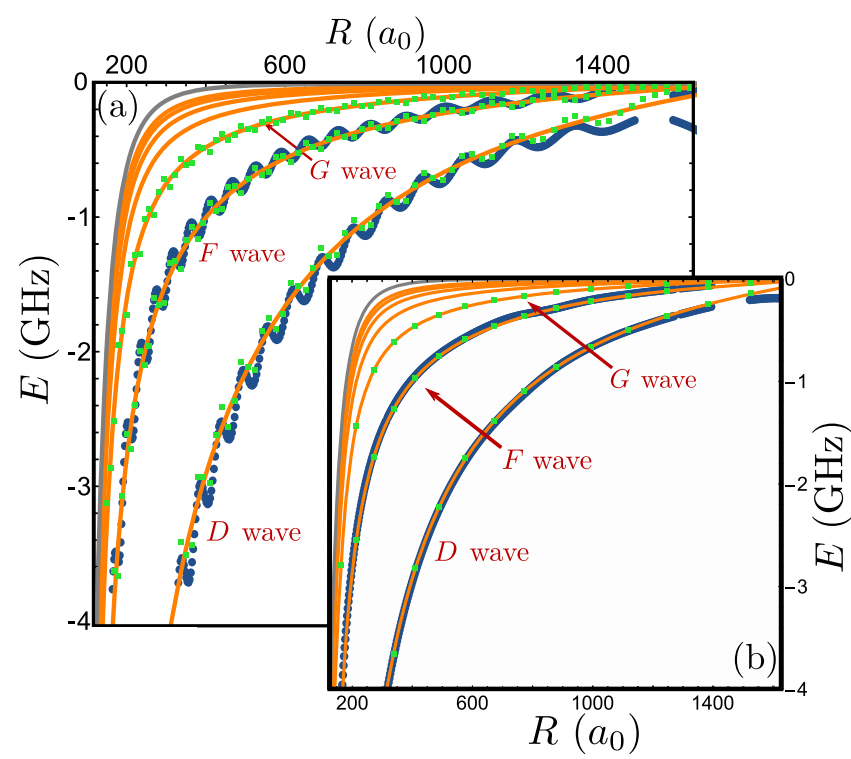

FIG. 2. The $L \geq 2$ PECs relative to the $n=30$ manifold for (a) $\Sigma$ and (b) $\Delta$ molecular symmetry using the BA phase shifts. The orange (blue) lines indicate the PECs within the ion-pair model (GLFT approach). The green squares denote the results of the numerical perturbation theory for a soft-core polarization potential. The polarization potential is shown in gray. We have set $\mu_{l}=0$ for this comparison.

the atom-ion potential (gray) as $L \rightarrow \infty$. As seen in butterfly ULRMs, the PEC oscillations disappear with increasing $M$ [30,31,54]. Figure 2(b) shows the smooth $\Delta$ PECs: the results of the GLFT method (blue) are in excellent agreement with the dressed ion-pair model (orange).

In general, the calculations of GLFT theory for $L \leq 1$ match those obtained by the Green's function method [44-46] and Omont's pseudopotential (see [49] for details). Note that these methods share a crucial approximation: the phase shifts are fully accumulated at the perturber, and thus the polarization potential is replaced with a zero-range $e-B$ interaction. The validity of this approximation breaks down near the classical turning point where the $e-B$ momentum vanishes, yielding divergent scattering volumes $-\tan \delta_{L}(k) / k^{2 L+1}$ for $L \geq 1$ [53]. This low-energy unphysical behavior could invalidate the molecular PECs, especially for $L \geq 2$. Therefore, the $L \geq 2$ PECs were numerically calculated by diagonalizing a divergence-free, soft-core polarization potential $V_{e-B}(\mathbf{r})=-(\alpha / 2)\left(\beta^{4}+|\mathbf{r}-\mathbf{R}|^{4}\right)^{-1}$, thus avoiding the use of the phase shifts altogether. In Fig. 2 the soft-core results (green squares) match the GLFT results (orange curves) for all $R<R_{c}$, beyond which the GLFT, and in fact all methods using phase shifts as external input, break down.

Figure 3 confirms the Rydberg character of the vibrational spectrum of the adiabatic Born-Oppenheimer dragonfly PECs, which we focus on as they are the deepest

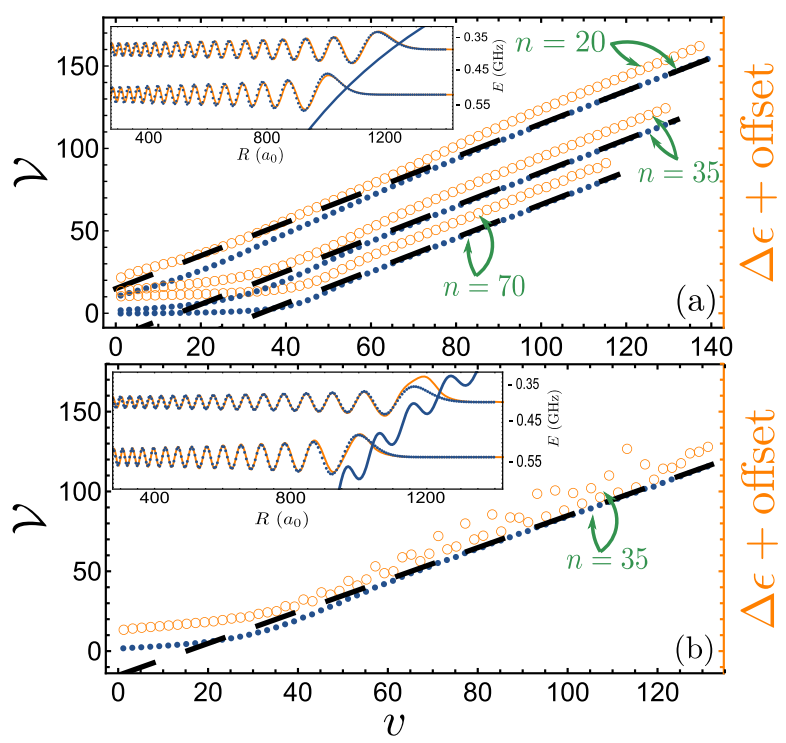

FIG. 3. The effective nuclear quantum number $\mathcal{V}$ (blue dots) and the rescaled difference of successive energy levels $\Delta \epsilon$ (orange circles) for the (a) $\Delta$ and (b) $\Sigma$ "dragonfly" molecular curves at different $n$ manifolds. The dashed lines correspond to linear fits of $\mathcal{V}$ with unity slope. The insets depict the nuclear wave function at two different eigenenergies evaluated either numerically (orange line) or by using fitted Coulomb wave functions (blue dots). Note that an arbitrary offset is added to $\Delta \epsilon$ for illustration purposes.

of the $L \geq 2$ PECs. Here, we remove any approximations on the phase shifts and use the exact, rather than the BA, $\delta_{L}$. We show results for fixed nuclear rotational quantum number $J=0$. The spectra, $\varepsilon_{v J}^{n L}$, are obtained numerically using a hard wall at $R=30 a_{0}$ which mimics the shortrange physics. Here, a more detailed theoretical description of the latter would only result in modified quantum defects $\eta_{J}$, maintaining the Rydberg characteristics of the $\varepsilon_{v J}^{n L}$.

Across several $n$ manifolds, Fig. 3(a) shows the effective nuclear quantum number $\mathcal{V}=\sqrt{R_{n L}^{\prime} /\left(\bar{\alpha}_{L} / n^{5}-\varepsilon_{v J}^{n L}\right)}$ (blue dots) for the $\Delta$ dragonfly PECs. The black dashed lines show the $\mathcal{V}=v-\eta_{J}$, derived from Eq. (5), where we have fitted $\eta_{J}$ to the numerical data. The evident linear dependence manifests a THRS. For high $n$ manifolds, i.e., $n=70$, the nuclear states for $v>50$ yield a Rydberg spectrum; deviations occur at $v<50$ due to the polarization potential. At low $n$ manifolds, i.e., $n=20$, nearly all the nuclear states form a Rydberg series since the Coulomb-like PEC dominates the polarization potential due to the relatively large effective charge. In general, at large $v$, the quantum numbers $\mathcal{V}$ agree well with the dashed black lines which correspond to Eq. (5) with a fitted nuclear quantum defect $\eta_{J}$.

Figure 3(a) also shows the rescaled difference of successive energy levels, $\Delta \epsilon=\left|2\left(\varepsilon_{v J}^{n L}-\varepsilon_{v-1 J}^{n L}\right) / R_{n L}^{\prime}\right|^{-1 / 3}$ (orange circles), which more sensitively probes the Rydberg character of the series. The linear dependence 
of $\Delta \epsilon$ on $v$ with unity slope indicates that the nuclear states possess the proper Rydberg energy scaling, i.e., $\Delta E \sim 1 / v^{3}$.

Figure 3(b) shows the same quantities as in 3(a) for the $\Sigma$ "dragonfly" PEC. In Fig. 2(a) the potential wells are too shallow to support localized bound vibrational states. Instead, they produce low-amplitude oscillations of $\mathcal{V}$ around the linear fit (black dashed lines) which are seen faintly on this scale. The $\Delta \epsilon$ values show this modulation more explicitly, highlighting the non-Rydberg nature of the corresponding spectrum. The insets in Fig. 3 illustrate the numerical nuclear wave functions (orange lines) at two different eigenenergies; the blue dots denote fits to the Coulomb wave functions. Evidently, in Fig. 3(a) the numerical and the fitted wavefunctions are in excellent agreement. In panel (b), deviations in the outermost lobes are observed due to the wells in the $\Sigma$ PEC, but the overall nodal pattern of the wave functions is determined by the dominant energy scale of the Coulomb-like potential.

The laboratory excitation of THRS will be similar to that of trilobitelike ULRMs, requiring three-photon excitation via admixture of the $n f$ quantum defect state of $\mathrm{Rb}$ or by using other, more favorable, quantum defect states in other atoms [6,48,55]. The nuclear quantum defects can be extracted by scanning the appropriate energy range to obtain a spectrum which can be fit to the Rydberg formula. The short-range physics at $R<30 a_{0}$, where molecular ion formation and other ultracold chemistry can occur, is beyond the scope of the present work, but it will affect the size of the nuclear quantum defects and, more importantly, the lifetime of the Rydberg states [16]. A firm upper limit on the lifetime is set by the unperturbed Rydberg atom's lifetime, which depends on $(n, l)$ quantum numbers and for the $n$ values considered in typical experiments can range from $1-100 \mu \mathrm{s}$. However, as in the HRS, the lifetimes will likely be substantially reduced by nuclear decay channels to $\sim 1-100 \mathrm{~ns}$. These lifetimes increase with both $v$ and $J ; J$ could be increased by applying a very weak electric field to create pendular states [56].

In summary, we have identified new vibrational states in ultralong-range molecules which form a trimmed heavy Rydberg series with very small Rydberg constant. The GLFT method for ULRM method enables us to accurately determine the highly excited underlying Coulomb-like PECs that stem from the electron's interaction with with the perturber. Although we focused on these new attributes, the effects of higher partial waves may contribute to more accurate theoretical spectra to compare with ongoing experimental efforts $[8,9,11,42,48]$. Finally, because of the generic character of the presented binding mechanism, we expect that similar trimmed Rydberg series, as described and analyzed here in the context of ULRMs, could occur in any system containing a Rydberg atom and a polarizable perturber, e.g., atoms with more complex structure, multiple atoms, excitons, or even large compounds like fullerines or nanodroplets.

We thank Chris H. Greene for useful comments and suggestions about the manuscript. M. T. E. is grateful to the Alexander von Humboldt stiftung for support through a postdoctoral fellowship. F. R. acknowledges the financial support by the U.S. Department of Energy (DOE), Office of Science, Basic Energy Sciences (BES) under Award No. DE-SC0012193. The numerical calculations have been performed using NSF XSEDE Resource Allocation No. TG-PHY150003.

"pgiannak@pks.mpg.de

"meiles@pks.mpg.de

robichf@purdue.edu

s rost@pks.mpg.de

[1] C. H. Greene, A. S. Dickinson, and H. R. Sadeghpour, Phys. Rev. Lett. 85, 2458 (2000).

[2] J. Shaffer, S. Rittenhouse, and H. Sadeghpour, Nat. Commun. 9, 1965 (2018).

[3] M. T. Eiles, J. Phys. B 52, 113001 (2019).

[4] C. Fey, F. Hummel, and P. Schmelcher, Mol. Phys. 118, e1679401 (2020).

[5] V. Bendkowsky, B. Butscher, J. Nipper, J. B. Balewski, J. P. Shaffer, R. Löw, and T. Pfau, Nature (London) 458, 1005 (2009).

[6] J. Tallant, S. T. Rittenhouse, D. Booth, H. R. Sadeghpour, and J. P. Shaffer, Phys. Rev. Lett. 109, 173202 (2012).

[7] B. J. DeSalvo, J. A. Aman, F. B. Dunning, T. C. Killian, H. R. Sadeghpour, S. Yoshida, and J. Burgdörfer, Phys. Rev. A 92, 031403(R) (2015).

[8] M. Deiß, S. Haze, J. Wolf, L. Wang, F. Meinert, C. Fey, F. Hummel, P. Schmelcher, and J. H. Denschlag, Phys. Rev. Research 2, 013047 (2020).

[9] F. Engel, T. Dieterle, F. Hummel, C. Fey, P. Schmelcher, R. Löw, T. Pfau, and F. Meinert, Phys. Rev. Lett. 123, 073003 (2019).

[10] H. Saßmannshausen, F. Merkt, and J. Deiglmayr, Phys. Rev. Lett. 114, 133201 (2015).

[11] J. L. MacLennan, Y.-J. Chen, and G. Raithel, Phys. Rev. A 99, 033407 (2019).

[12] K. S. Kleinbach, F. Engel, T. Dieterle, R. Löw, T. Pfau, and F. Meinert, Phys. Rev. Lett. 120, 193401 (2018).

[13] T. Schmid, C. Veit, N. Zuber, R. Löw, T. Pfau, M. Tarana, and M. Tomza, Phys. Rev. Lett. 120, 153401 (2018).

[14] J. D. Whalen, R. Ding, S. K. Kanungo, T. C. Killian, S. Yoshida, J. Burgdörfer, and F. B. Dunning, Mol. Phys. 117, 3088 (2019).

[15] M. Schlagmüller, T. C. Liebisch, H. Nguyen, G. Lochead, F. Engel, F. Böttcher, K. M. Westphal, K. S. Kleinbach, R. Löw, S. Hofferberth, T. Pfau, J. Pérez-Ríos, and C. H. Greene, Phys. Rev. Lett. 116, 053001 (2016).

[16] M. Schlagmüller, T. C. Liebisch, F. Engel, K. S. Kleinbach, F. Böttcher, U. Hermann, K. M. Westphal, A. Gaj, R. Löw, S. Hofferberth, T. Pfau, J. Pérez-Ríos, and C. H. Greene, Phys. Rev. X 6, 031020 (2016). 
[17] A. Gaj, A. T. Krupp, J. B. Balewski, R. Löw, S. Hofferberth, and T. Pfau, Nat. Commun. 5, 4546 (2014).

[18] F. Engel, T. Dieterle, T. Schmid, C. Tomschitz, C. Veit, N. Zuber, R. Löw, T. Pfau, and F. Meinert, Phys. Rev. Lett. 121, 193401 (2018).

[19] J. D. Whalen, S. K. Kanungo, R. Ding, M. Wagner, R. Schmidt, H. R. Sadeghpour, S. Yoshida, J. Burgdörfer, F. B. Dunning, and T.C. Killian, Phys. Rev. A $\mathbf{1 0 0}$, 011402(R) (2019).

[20] R. Schmidt, H. R. Sadeghpour, and E. Demler, Phys. Rev. Lett. 116, 105302 (2016).

[21] R. Schmidt, J. D. Whalen, R. Ding, F. Camargo, G. Woehl, S. Yoshida, J. Burgdörfer, F. B. Dunning, E. Demler, H. R. Sadeghpour, and T. C. Killian, Phys. Rev. A 97, 022707 (2018).

[22] F. Camargo, R. Schmidt, J. D. Whalen, R. Ding, G. Woehl, S. Yoshida, J. Burgdörfer, F. B. Dunning, H. R. Sadeghpour, E. Demler, and T. C. Killian, Phys. Rev. Lett. 120, 083401 (2018).

[23] Y. Ashida, T. Shi, R. Schmidt, H. R. Sadeghpour, J. I. Cirac, and E. Demler, Phys. Rev. Lett. 123, 183001 (2019).

[24] J. Sous, H. R. Sadeghpour, T. C. Killian, E. Demler, and R. Schmidt, Phys. Rev. Research 2, 023021 (2020).

[25] E. Fermi, Il Nuovo Cimento 11, 157 (1934).

[26] A. Omont, J. Phys. (Paris) 38, 1343 (1977).

[27] K. S. Kleinbach, F. Meinert, F. Engel, W. J. Kwon, R. Löw, T. Pfau, and G. Raithel, Phys. Rev. Lett. 118, 223001 (2017).

[28] D. Booth, S. T. Rittenhouse, J. Yang, H. R. Sadeghpour, and J. P. Shaffer, Science 348, 99 (2015).

[29] W. Li, T. Pohl, J. M. Rost, S. T. Rittenhouse, H. R. Sadeghpour, J. Nipper, B. Butscher, J. B. Balewski, V. Bendkowsky, R. Löw, and T. Pfau, Science 334, 1110 (2011).

[30] E. L. Hamilton, C. H. Greene, and H. R. Sadeghpour, J. Phys. B 35, L199 (2002).

[31] M. I. Chibisov, A. A. Khuskivadze, and I. I. Fabrikant, J. Phys. B 35, L193 (2002).

[32] T. Niederprüm, O. Thomas, T. Eichert, C. Lippe, J. Pérez-Ríos, C. H. Greene, and H. Ott, Nat. Commun. 7, 12820 (2016).

[33] M. O. Vieitez, T. I. Ivanov, E. Reinhold, C. A. de Lange, and W. Ubachs, Phys. Rev. Lett. 101, 163001 (2008).

[34] E. Reinhold and W. Ubachs, Phys. Rev. Lett. 88, 013001 (2001).
[35] E. Reinhold and W. Ubachs, Mol. Phys. 103, 1329 (2005)

[36] A. Kirrander, S. Rittenhouse, M. Ascoli, E. E. Eyler, P. L. Gould, and H. R. Sadeghpour, Phys. Rev. A 87, 031402(R) (2013).

[37] S. Mollet and F. Merkt, Phys. Rev. A 82, 032510 (2010).

[38] S. Markson and H. Sadeghpour, J. Phys. B 49, 114006 (2016).

[39] M. Peper and J. Deiglmayr, J. Phys. B 53, 064001 (2020).

[40] F. Hummel, P. Schmelcher, H. Ott, and H. R. Sadeghpour, New J. Phys. 22, 063060 (2020).

[41] P. Giannakeas, M. T. Eiles, F. Robicheaux, and J.-M. Rost, Phys. Rev. A 102, 033315 (2020).

[42] M. T. Eiles and C. H. Greene, Phys. Rev. A 95, 042515 (2017).

[43] C. Fey, M. Kurz, P. Schmelcher, S. T. Rittenhouse, and H. R. Sadeghpour, New J. Phys. 17, 055010 (2015).

[44] C. H. Greene, E. L. Hamilton, H. Crowell, C. Vadla, and K. Niemax, Phys. Rev. Lett. 97, 233002 (2006).

[45] E. L. Hamilton, Photoionization, photodissociation, and long-range bond formation in molecular Rydberg states, Ph.D. thesis, University of Colorado, 2002.

[46] A. A. Khuskivadze, M. I. Chibisov, and I. I. Fabrikant, Phys. Rev. A 66, 042709 (2002).

[47] M. Tarana and R. Curík, Phys. Rev. A 99, 012708 (2019).

[48] M. T. Eiles, Phys. Rev. A 98, 042706 (2018).

[49] See the Supplemental Material at http://link.aps.org/ supplemental/10.1103/PhysRevLett.125.123401 for more discussion on the electron-alkali phase shifts, the dipole moments of the dragonfly molecules, the derivations using Omont's $d$-wave pseudopotentials and the dressed ion-pair model. The Supplemental Material includes Ref. [50].

[50] M. Marinescu, H. R. Sadeghpour, and A. Dalgarno, Phys. Rev. A 49, 982 (1994).

[51] F. T. Smith, Phys. Rev. 118, 349 (1960).

[52] V. M. Borodin and A. K. Kazansky, J. Phys. B 25, 971 (1992).

[53] N. Y. Du and C. H. Greene, Phys. Rev. A 36, 971 (1987); see also the erratum,36, 5467(E) (1987).

[54] P. Schmelcher, C. Fey, M. Eiles, and F. Hummel, J. Phys. B 53, 084001 (2020).

[55] M. T. Eiles and C. H. Greene, Phys. Rev. Lett. 115, 193201 (2015).

[56] J. M. Rost, J. C. Griffin, B. Friedrich, and D. R. Herschbach, Phys. Rev. Lett. 68, 1299 (1992). 